\title{
Les propriétés de figement dans le profil combinatoire des verbes défectifs seoir et messeoir
}

\section{The Combinatory Profile of Defective Verbs seoir and messeoir: the Stability and Fixedness}

\author{
JANA BRŇÁKovÁ [jana.brnakova@osu.cz] \\ Ostravská univerzita, République tchèque
}

\begin{abstract}
RÉSUMÉ
Le verbe défectif seoir et son préfixé antonymique messeoir sont, du point de vue synchronique, considérés en tant que défectifs, c'est-à-dire des verbes dont les taux de fréquence sont négligeables et dont les paradigmes de conjugaison restent incomplets en français contemporain. À l'aide de l'analyse de 61 cooccurrences attestées du verbe seoir dans un ensemble de 152 textes au nombre de 11176247 mots, le moteur de recherche de la base textuelle Frantext renvoie 10 formes conjuguées du verbe en question, dont 37,7 $\%$ sont représentées par les troisièmes personnes. Ceci confirme le postulat portant sur la productivité de ce verbe dans le stock lexical du français, dans la mesure où il est susceptible de subir des actualisations de leurs configurations actancielles. Par contre, le lexème messeoir n'est attesté dans aucun des textes postérieurs à l'an 2000 dans le Frantext. À cause de cette abscence d'occurrences, il est possible de considérer ce verbe en voie de disparition du fond actif du lexique français.
\end{abstract}

\section{MOTS CLÉS}

verbes défectifs; profil combinatoire; collocation; figement; combinabilité; valence; frantext; TLFi

\begin{abstract}
The French verb seoir and its equivalent prefix-based antonym messeoir are from a synchronic perspective to the contemporary French word-stock treated as defective verbs, whose frequency of occurrence is rather rare and whose limited range of verb forms results in a paradigmatically incomplete conjugation. The data analysis of 61 verb phrases of seoir in 152 various texts from the Frantext corpus comprising $11,176,247$ running words shows the occurrence of 10 non-finite forms, out of which $37.7 \%$ have the form of the third person singular/plural. The outcomes clearly demonstrate that some verb forms are still popular in the language and keep their combining power or valence. In contrast, the lexeme messeoir does not occur at all in the corpus data after the year 2000. Based on the zero occurrence it may be argued that the verb has been gradually moving to the periphery of the contemporary word-stock.
\end{abstract}

\section{KEYWORDS}

defective verbs; combinatory profile; collocation; stability; combinability; valence; Frantext corpus

REÇU 2017-03-07 ; ACCEPTE 2017-05-25 


\section{Introduction}

Les verbes défectifs sont, d’une manière générale, définis en tant que verbes dont le paradigme de conjugaison est devenu au fil du temps incomplet. Dans l'usage du français contemporain, il n'en perdure que quelques modes, temps ou personnes, qui restent usités au sein de groupements de mots liés par un certain degré de figement, qui va progressant des collocations aux parémies, pour lesquelles la fixité est considérée comme la plus complète. Dans les collocations, les constituants lexicaux gardent leur sens propre (en faire accroire à qqn), ce qui assure à cette séquence figée une lecture compositionnelle. La lexie pivot peut cependant également subir une spécialisation de signification, comme en est le cas du verbe apparoir, qui ne s'avère plus attesté que sous la $3^{\mathrm{e}}$ personne du singulier de l'indicatif présent dans le registre juridique ou littéraire du français (cf. Le Petit Robert 2014) : ainsi qu'il appert des dépositions des témoins. Quelques-uns des verbes défectifs restent figés dans le cadre des énoncés phrastiques, sous forme de proverbes ou dictons qui découlent des expériences, de longue durée parfois, transmises par des générations, au sein d'une communauté langagière. À titre d'exemple on peut citer la parémie Oignez vilain, il vous poindra; poignez vilain, il vous oindra, qui contient deux verbes considérés comme défectifs.

Certains linguistiques sont même persuadés que, si ces verbes en question restent usités, cela ne sera que dans le cadre de ces groupements de mots figés : " la plupart des verbes défectifs sont condamnés à disparaître ou du moins à ne subsister que dans des locutions toutes faites. Déjà, parmi les formes que la tradition maintient pieusement dans les grammaires, il en est beaucoup que la langue parlée ignore absolument et auxquelles la langue écrite ne conserve qu'artificiellement un certain souffle de vie» (Grevisse $2001: 1234$ ).

Le rôle de l'usage dans la formation des entités lexicalisées à base des verbes défectifs est décisif. Dans sa dimension diachronique, le processus de l'usage aboutit à la fixation de l'emploi de quelques modes, temps ou personnes de cette catégorie de verbes en question qui ont cessé de fonctionner dans toute la complexité du paradigme susceptible de se soumettre aux règles combinatoires de la syntaxe libre ou vivante (Schapira 1999 : 8). Grâce à la répétition de groupements soudés allant de l'unité indissoluble de deux ou plusieurs mots jusqu’à des énoncés phrastiques, l'usage de verbes défectifs a acquis à travers le temps un nouveau statut avoisinant avec la fonctionnalité des unités phraséologiques. La reprise automatique de certaines formulations stéréotypiques contenant des verbes défectifs pourrait ainsi éviter l'étape finale de la voie entamée de leur disparition totale en français de nos jours.

Cette stabilité de l'usage de verbes défectifs au sein des groupements de mots figés confirme le postulat de Mejri (2008 : 121) que « c'est par le biais du retour de la parole sur la langue que le renouvellement du système est assuré ». Si nous concevons le figement comme une reprise de formation stable dotée d'une signification fixe, approuvée par un usage statistiquement vérifiable, nous avons ici recours à un parcours inverse entre les anciennes dichotomies saussuriennes langue/parole. Ce n'est qu'à la suite d'une attestation vérifiée que les pratiques discursives des séquences figées sont intégrées dans le corps de l'article des entrées des dictionnaires et ainsi codifiées.

Le présent article s'efforce, avant tout, d'attester de la vivacité de séquences fixes résultant du figement de l’emploi des verbes étudiés (seoir et son préfixé antonymique messeoir) à l'aide 
d'une confrontation du codage lexicographique (Le Robert 2014, TLFi en ligne, Larousse 2009 en ligne) avec les résultats obtenus d'une source électronique de données statistiquement vérifiables - Frantext.

\section{Définition du corpus de travail}

Pour pouvoir analyser les propriétés des figements du profil combinatoire des verbes seoir et messeoir, nous avons opté pour Frantext, le grand corpus informatisé accessible (après abonnement) grâce à la plateforme $w w w$.frantext.fr. Visant à décrire les aspects des combinaisons lexico-syntaxiques de deux verbes en français contemporain, nous avons limité la taille du corpus aux textes postérieurs à l'an 2000 et nous avons requis que le contexte soit restreint à une même phrase. Sur un ensemble de 152 textes, au nombre de 11176247 mots, le moteur de recherche a trouvé 65 valences actancielles du profil syntaxique de la lexie pivot seoir, mais par contre il n'a trouvé aucune attestation pour le verbe intransitif indirect messeoir. Pour la visualisation des résultats, nous avons choisi le formulaire de paramétrage par paquet de 50 attestations de KWIC (key word in context). Ce mode de présentation nous a permis d’opérer un tri sélectif des cooccurrents et de les reporter par la suite dans les catégories saisies qui se répètent.

Pour éviter des usages uniques de chaînes de caractères répondant au critère imposé, nous avons recouru aux éléments bibliographiques indiquant leur origine. Si la chaîne a été retrouvée dans l'identifiant unique dans la même cote à plusieurs reprises, nous l'avons considérée en tant qu'aléatoire, due au génie inventif de l'auteur en question.

Dans la deuxième étape de nos recherches, nous avons procédé à une analyse comparative des résultats obtenus via Frantext avec le profil lexical de la base dans les dictionnaires faisant autorité (voir ci-dessous, Le Robert 2014, TLFi et Larousse 2009 en ligne).

Pour clarifier les analyses effectuées, ainsi que nos points de vue théoriques, nous avons inséré dans le corps de l'article des extraits dépouillés de la base textuelle Frantext qui visent à éviter les paragraphes d'explications redondants et également à fournir les illustrations nécessaires. Les exemplifications empruntées à Frantext sont étayées par une indication bibliographique globale, renvoyant au site officiel $w w w$.frantext.fr et diffèrent des autres citations standardisées par leur format, qui respecte systématiquement l'unicité de la démarche et de la méthodologie imposées par les auteurs de ce corpus électronique.

\section{Extraction et tri de cooccurrents}

Afin de saisir les cooccurrences figées du verbe seoir, nous avons effectué plusieurs requêtes dans Frantext au mois de septembre 2014. Lors de la première, nous avons recherché toutes les formes conjuguées du verbe en question à l'aide de \&cseoir. Nous résumons le tri des résultats, sélevant à 65 cooccurrences, obtenus dans le graphique suivant : 


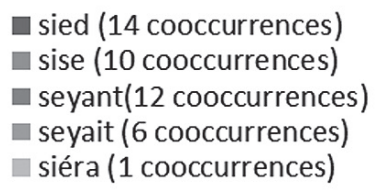

Après une catégorisation de discrimination, nous avons procédé à des recherches sélectives portant sur chaque forme, mode et temps, que nous élucidons dans ce qui suit en fonction du taux de fréquence des cooccurrences repérées.

\section{L'infinitif seoir}

L'infinitif seoir, issu du verbe latin sedere, a fini en français par la formation de deux valeurs, répertoriées dans les dictionnaires sous deux entrées différentes. La première (désormais seoir 1) comporte l'indication qu'il s'agit d'un mot vieux « de l'ancienne langue qui est peu compréhensible de nos jours et jamais employé, sauf par effet de style » (cf. Le Petit Robert 2014). Le contenu de cet article est limité à une seule signification : " être assis ». En faisant référence à Richelet (1680), le Trésor informatisé de la langue française (TLFi) indique même qu'avec cet effet de sens, le verbe en question est déjà qualifié de vieilli au XVII ${ }^{e}$ siècle et qu'en sa place on préfère le verbe s'asseoir. Les deux ressources lexicographiques (Le Petit Robert 2014 et le TLFi) s'accordent sur la constatation qu'au sens de « être assis » ce verbe est le plus souvent usité au participe présent (séant) et au participe passé (sis, sise).

La deuxième acception du verbe polysémique (désormais seoir 2), dans la nomenclature alphabétique qui suit, donne matière à une exemplification plus large dans des contextes attendus après une information générale sur cette lexie, également désignée vieillie ou littéraire, qui signifie « convenir, aller ». Celle-ci, même si elle s'encadre dans le même modèle de conjugaison, atteste d'un éventail plus large de son emploi temporel (indicatif du présent, de l'imparfait, futur simple, conditionnel présent) dont on confirme uniquement la vivacité des troisièmes personnes du singulier et du pluriel. Reste également en usage le participe présent et l'adjectif 
séant, -ante, ayant son entrée à part entière dans les dictionnaires, avec sa variante moderne seyant, -ante, due aux formes analogiques de l'imparfait seyait.

\subsection{Le présent de l'indicatif (il sied)}

Conformément à l'information sur le signe seoir dans les deux sources lexicographiques indiquées ci-dessus, le moteur de recherche n'a trouvé que quatorze apparitions de la troisième personne de l'indicatif du présent au singulier (sied). Ceci confirme le bien-fondé de son statut de verbe défectif. Dans tout l'ensemble des cooccurrences trouvées, les compléments d'objet sont régis par la préposition $\grave{a}$, y compris toutes les manifestations morphologiques apparentées à l'évolution de la langue française (par ex. : au, auquel, etc.). Nous n'avons trouvé dans les contextes tirés de Frantext qu'un seul emploi impersonnel, il sied de + infinitif : ... il ne me sied pas de les voir... Nous n'avons attesté dans notre corpus aucune apparition d'enchaînement de l'infinitif à l'aide de la préposition que, suivie par le subjonctif.

En analysant la valence de cette forme verbale, nous avons repéré neuf combinaisons réalisées avec les actants animés humains, exprimés dans six exemplifications par les pronoms. Les autres actants, soumis à la combinabilité libre des intentions parfois momentanées des écrivains, désignent des substantifs inanimés ou des notions abstraites : lâge, un camion, le noir, le chienlit, au teint, l'indigence, le romantisme, petites mortes améthystes, au réveil, au coucher ou au réveil, la comparaison, un programme. N'étant pas de fréquence statistiquement significative, ces groupements de mots sont interprétables comme usuels, résultant de la conjonction des contraintes grammaticales et des portées référentielles propres au système langagier du français.

Cette forme verbale atteint le taux de fréquence le plus élevé dans les extraits tirés de Frantext, à part égale avec le participe passé (sis) correspondant à la première acception du verbe polysémique en question, dont l'usage au sens de « situé » est maintenu en français contemporain dans le domaine littéraire ou dans celui du droit.

\subsection{L'adjectif seyant}

À la différence de l'adjectif séant, sa variante moderne seyant, formée à l'instar de l'imparfait (cf. Le Petit Robert 2014), est élucidée par des significations coïncidant parfaitement dans les deux sources lexicographiques (cf. Le Petit Robert et le TLFi) : « qui convient ", « qui donne un aspect agréable à une personne ou à une chose », ou tout simplement " qui va à quelqu'un ». En analysant l'aspect sémique de ces valeurs, nous pouvons présupposer que cet adjectif entrera dans le voisinage de mots constituant un vaste champ lexical décrivant l'aspect du visage. Ceci se trouve confirmé par huit cooccurrences observées dans les extraits tirés de Frantext. Chacune d'elles contient au moins un sème avoisinant avec des propriétés vestimentaires ou des apparences au sens large : 
R158 - LE CLÉZIO Jean-Marie Gustave, L'Africain, 2004, p. 50

L'Africain

Les lorgnons n’étaient déjà plus très courants à cette époque. Peut-être qu'à Nice quelques vieux de la vieille avaient conservé cet accessoire, que j'imagine seyant parfaitement à d'anciens officiers russes de l'armée impériale, arborant moustache et favoris, ou bien inventeurs ruinés qui fréquentaient mes « tantes ».

R095 - HAVET Mireille, Journal 1919-1924, 2005, p. 197

JOURNAL 1921

Paris est d'une admirable couleur de cataclysme, d’orage et de cuivre. Les Champs-Elysées, le soir, sont une fournaise saumon. Jamais fard plus seyant n'exista pour ces femmes qui traversent vers le crépuscule, la place admirable de la Concorde, où, déjà, les grands candélabres dessinent un nouveau ciel, à rebours de l'autre et

E092 - GARAT Anne-Marie, Pense à demain, 2010, p. 112

V Samedi 7 septembre

Efforçait de reprendre sa dignité, jetant au passage un dernier coup d'œil au miroir pour y évaluer sa bienséante et flegmatique image, l'effet de sa robe jaune, safran clair, très seyant sur sa peau mate, avec ses yeux bruns ; noisette, disait-on pour la flatter. Elle avait toujours su que cétait un marron banal, une couleur commune à côté de ceux de sa soeur,

E092 - GARAT Anne-Marie, Pense à demain, 2010, p. 367

XIII Mardi 12 novembre

des mines, des poses aguichantes, se laisser tripoter, se promettre, se dérober, appâter sans rien lâcher. Avant la bague au doigt : bas les pattes. Donnant, donnant. C'est très seyant, ces fuseaux de ski, casaque en shetland, bonnet interplanétaire en Crylor et les bottes fuselées, en mouton retourné. Pulls fins, deuxième peau sous l'anorak style cosmonaute

E056 - DOUBROVSKY Serge, Un homme de passage, 2011, p. 240

IMAGES

Je me souviens, la scène date du tout début de la décennie soixante-dix. Rachel, très jolie, porte une superbe robe de laine bleue avec un col roulé fort seyant. C'était l'époque où elle croyait que ma famille serait bientôt sa famille. Illusion qu'elle a mis du temps à perdre. Moi aussi, du reste.

E084 - JENNI Alexis, L’Art français de la guerre, 2011, p. 473

COMMENTAIRES VI Je la voyais depuis toujours, mais jamais je n'aurais osé lui parler. Ce fut aussi des dames mûres, qui tournaient dans les rues en uniforme peu seyant, à la recherche des mal-garés ; elles sermonnaient les ados qui l'été plongeaient dans la Saône, en affirmant qu'elles n'iraient pas les chercher, et elles s'engueulaient avec

S731 - BÉNABOU Marcel, Écrire sur Tamara, 2002, p. 239

XIX Troisième année

Tout ce que tu mettais derrière ce mot, c'était une image puérilement épurée. Tu voyais Tamara, portant sur le visage un air de langueur et de fragilité particulièrement seyant, passer ses journées à lire et à rêver dans une chambre toute blanche, sur un large fauteuil laqué.

E119 - GAVALDA Anna, Ensemble, c'est tout, 2004, p. 468

QUATRIÈME PARTIE, 13

- Il est pas mal, non?

$-\ldots$

- Franchement je m’attendais à pire... Il est sport comme modèle... Noir comme ça, c'est chic...

- Ben voyons... Dis-moi qu'il est seyant pendant que t'y es !

Une seule fois, cet adjectif apparaît nominalisé, assumant la fonction de substantif concret dont le KWIC (key word in context) contient un anthroponyme hautement symbolique pour les habitudes collectives en matière d'habillement et se situe dans une zone d'inclusion avec le champ lexical de vêtement précisé ci-dessus : 
E162 - GARAT Anne-Marie, Lenfant des ténèbres, 2008, p. 472

XVI

Mais le secret de tout cela, l'astuce vraiment géniale : le biais ! La fluidité élastique du biais fauché à Coco. Il n’y a pas brevet, ma belle. Le seyant du biais qui épouse et drape, dissimule, allonge, dont l'oblique se façonne à volonté, surtout s'il joue du bicolore, camaieu de gris, de vert.

En analysant d'autres voisinages du signe seyant, nous sommes parvenue à constater que les autres combinaisons relèvent de la combinabilité facultative qui donne la possibilité de choix de combiner cette lexie avec d'autres conformément aux règles grammaticales du code langagier. Cet adjectif s'applique à des substantifs concrets :

R126 le kola en astrakan fin, marron, gris ou blanc, si seyant, doit en enlever aux Persans. Les marchands de

Il constitue une expansion accompagnant des notions abstraites ou les noms d'action qu'il détermine :

R217 développement " ou " co-développement », il est seyant, élégant même. La réalité l’est moins, qui mérite E086 les porter à l'envol déserteur. Leur ramage est seyant puisqu'ils nous ont plumées. Car engrainée ou

Fonctionnant en tant qu'adjectif, il nous était nécessaire de lancer une requête complémentaire sur son genre féminin et sur son nombre (\&mseyant), car les deux variabilités étaient absentes des cooccurrences trouvées par le moteur de recherche pour le verbe en question. Ainsi le nombre de co-apparitions s'est-il augmenté de trois nouvelles combinaisons qui restent encadrées dans la même zone d'intersection des valeurs sémantiques indiquées. Deux signifiants au pluriel ont, dans leurs voisinages, un mot emprunté à l'anglais pour désigner une veste en flanelle (blazers) et une expression qui lui est dans un rapport d'hyperonymie - les combinaisons de vêtements. Le troisième emploi réfère également à l'aspect du visage :

$\mathbf{R} 120$ avec la mine qu'il arborait aujourd'hui, une autre couleur aurait été...moins seyante . Sonna plusieurs fois

Ayant une valeur sémantique qui équivaut à l'addition de l'acception de chaque élément lexical, les combinaisons de lexies ci-dessus analysées sont pourvues du statut de collocations décodables à l'aide de la signification de leurs constituants et peuvent par la suite être interprétées, conformément à la taxinomie de Tutin et Grossman (2002:12), comme transparentes.

\subsection{La lexie pivot séant}

Les valeurs sémantiques du signe lexical séant sont clarifiées dans les corps des articles dictionnairiques par trois acceptions majeures qui reflètent trois catégories grammaticales distinctes. Le participe présent, ou l'adjectif, du verbe au sens de seoir 1 sont employés dans la terminologie héraldique pour désigner la position assise des animaux représentés. Ils peuvent également former des collocatifs en combinaison avec les bases sémantiques indiquant des personnes 
siégeant dans une assemblée, ou même suite au processus du changement de sens de type métonymique, l'assemblée elle-même tenant séance. L'autre acception de cette unité lexicale correspond au verbe seoir 2. La combinabilité de cette lexie au sens de quelque chose ou quelqu'un " qui sied », qui « est admis comme convenable » ou qui " va à quelqu'un », est relativement large et illustrée par plusieurs exemplifications dans les œuvres lexicographiques. Considéré en tant que mot dont la productivité pour former des syntagmes en français est assez importante, aucune apparition n'a pourtant été trouvée par le moteur de recherche au sein de l'ensemble des textes postérieurs à l'an 2000 dans le Frantext. Ce mot y figure uniquement cinq fois comme substantif. Sur cet ensemble de cinq occurrences dans notre corpus, le participe présent substantivé, correspondant à seoir 1, forme quatre fois une collocation avec la préposition antéposée sur, suivie par le prédéterminant possessif son, ce qui constitue une cooccurrence lexicale statistiquement significative et attribue au syntagme le statut de collocation régulière. Les extraits tirés de Frantext apportent ainsi des éléments de preuve en montrant ce qu'affirment par ailleurs les dictionnaires. La lexie en question apparaît une fois dans le voisinage de la préposition sous, marquant la position de complément qui domine :

R146 périlleux efforts, éreintant deux hommes sous son séant , dessinait sur la face du père Louis le sourire

\subsection{Les participes passés (sis, sise, sises)}

Le participe passé du masculin du singulier (sis) atteint dans notre corpus le taux de fréquence le plus élevé (14 cooccurrences), de même que la troisième personne du présent de l'indicatif. En analysant les contextes typiques de ce sémion, nous pouvons constater sans réserve que le plus grand nombre de ses cooccurrents (10) désigne un lieu (à l'extrémité de l'objet oblong, dans son écrin de frondaisons séculaires, au 137 de la Müllerstrasse, dans une maison du XIXe siècle, à lautre bout de la rue, au premier étage, le long d'un aimable ruisseau, en face de la FLA, dans un bois de pins, au Kufurstendam). Cette attestation d'une pratique discursive sert d'explication à la persistance de cette formation stable dotée d'une signification fixe ("situé à ») dans les dictionnaires de langue (voir supra L'infinitif).

La prédominance des actants désignant les lieux, dans les configurations reflétant le codage conventionnel du participe sis, se prête à être confirmée par dix autres cooccurrences du genre féminin du participe en question. Dans les dix cas, le participe passé au féminin précède ou suit une indication d'orientation spatiale : à Johnsbury, la petite ville, au-delà de la Garonne, rue du Château, entre les voies du chemin de fer, rue du Lavoir, World Trade Center, au 36 rue de Meudon, au fond d'une combe, un peu plus bas que la piscine. Le seul emploi du genre féminin au pluriel, attesté dans le livre de Gérard Genette, entraîne un effet ironique :

R210 - GENETTE Gérard, Bardadrac, 2006, p. 417

des raviolis aux épinards que servait le père Milo (ou Millo ?) sur la terrasse de son auberge, à l'entrée de ce village de Peillon, dans l'arrière-pays niçois, le plus perché des villages perchés, que l'un de ses fidèles ajustement décrit comme un « vaisseau pétrifié par un mystérieux embargo mythologique ». Je me souviens aussi de l'Hôtel du Pic d'Anie, à

Lescun, où il fallait sortir pour atteindre les toiletten, sises un peu plus loin dans la (même) rue. Et de sa vue grisante sur le dit pic. 
Cela confirme encore une fois la thèse selon laquelle le paradigme de conjugaison de ce verbe n'est pas complètement éteint et qu'il se prête à former des syntagmes visant parfois les effets humoristiques.

\subsection{L'imparfait de l'indicatif}

Ce ne sont que deux personnes de l'imparfait de l'indicatif qui sont attestées dans l'ensemble de 152 textes, au nombre total de 11176247 mots : la troisième personne du singulier et la troisième personne du pluriel (seyait, seyaient). Ceci valide la conformité des descriptions lexicographiques du verbe défectif seoir 1 avec son usage réel à l'écrit (voir supra), selon lesquelles ce ne sont plus que ces deux personnes qui se maintiennent vivantes dans le stock lexical du français contemporain.

Le singulier est représenté dans notre corpus tiré de Frantext par six cooccurrences, dont trois ont été repérées dans la même œuvre d'Anne-Marie Garat, Une faim de loup : Lecture du Petit Chaperon rouge, publiée en 2004, où il a pour sujet des dénominations qui sont dans un rapport emblématique avec le titre : un petit chaperon rouge et ce couvre-chef. Une fois, cette forme verbale est en voisinage avec le substantif chemin que l'héroïne principale doit entreprendre.

Trois autres compléments d’objet indirect sont enchaînés à l'aide de la préposition $a$, impliquée par les règles opérationnelles de la syntaxe française. Deux actants identiques (à son teint) dans ces apparitions sont sémantiquement similaires avec les collocatifs de l'adjectif seyant ci-dessus analysé. Une fois, cette préposition précède l'actant, désignant le fait de se trouver en contact - rencontre.

La troisième personne du pluriel de l'indicatif de l'imparfait n'apparaît qu'une seule fois au sein de tous les textes postérieurs à l'an 2000 compris dans la base textuelle Frantext. Le verbe y suit les sujets désignant l'ornement d'une personne sous la forme du label et de la signature qui l'embellissent et lui ajoutent un certain caractère.

\subsection{Le futur simple (il siéra)}

Une seule exemplification a été trouvée par le moteur de recherche pour la troisième personne du singulier du futur simple. Précédé par la conjonction comparative comme, le syntagme contenant la forme verbale en question est inséré dans la proposition complexe sous forme de l'incise. Les deux autres actants antéposés ( $i$ l et vous) constituent des collocatifs impliquant des traits sémantiques communs avec le paradigme collocationnel du verbe seoir 2. Comme il vous siéra est une combinaison où tous les composants lexicaux ne perdent pas leur sens propre et l'acception de cette séquence figée est par la suite dotée de la valeur compositionnelle. Il est possible de décoder le sens de ce syntagme à l'aide de la signification de ses éléments constituants. Cette collocation revêt suffisamment les traits caractéristiques nécessaires pour qu’elle puisse être également rangée dans la catégorie des collocations transparentes de la classification typologique de Tutin et Grossman. ${ }^{1}$

1 (Tutin et Grossman 2002 : 12) Les collocations transparentes sont celles qui ont « un sens interprétable » - (grièvement blessé), donc décodable à l'aide de leurs éléments constituants. Cette catégorie reflète le mieux le caractère du semi-figement si propre au phénomène collocatif. 


\subsection{Le conditionnel présent (siérait)}

De même que dans le cas du futur simple, le conditionnel présent n'est attesté que par une seule combinaison réalisée au sein de tous les textes soumis aux critères de la définition de notre corpus de travail. La troisième personne du singulier apparaît dans le voisinage du sujet inanimé, désignant le processus au cours duquel on exige des éclaircissements ou des justifications sur les intentions ou sur la conduite de quelqu'un. Employée dans la construction négative, un peu d'explication est envisagé comme un acte non convenable au moment donné dans l'assertion tirée de Frantext pour illustrer le KWIC (le key word in context). Étant donné que ce groupement de mots est une exemplification isolée, qui n'est pas statistiquement significative (une seule apparition), il relève de la combinabilité libre, qui reste régie par les règles opérationnelles de la syntaxe libre du français.

\subsection{Le verbe messeoir}

Répertorié aussi parmi les verbes défectifs (Grevisse 2001 : 1245), la description de l'entrée messeoir contient, dans les dictionnaires (cf. Le Petit Robert 2014, TLFi et Larousse 2009 en ligne), l'indication qu'il s'agit d'un verbe qui s'emploie surtout en français écrit et élégant : à la forme négative et à la troisième personne du présent, de l'imparfait, du futur de l'indicatif et du conditionnel présent. Ce verbe intransitif indirect a été dérivé de seoir à l'aide du préfixe d’origine germanique mes- et est remplacé dans l'usage par des synonymes plus courants (ne pas convenir, nêtre pas séant). L'exemplification de ce verbe est la plus complète dans le TLFi, à la différence du Petit Robert 2014 où la description de l'entrée n'occupe que trois lignes complètes. Le TLFi résume le fonctionnement de cette unité lexicale dans trois paragraphes respectifs. Le premier porte sur le profil syntaxique prototypique avec l'emploi privilégié du complément prépositionnel à : ne pas messeoir à qqn ou qqc, doté de la signification " ne pas convenir ; n'être pas séant ». Le suivant se focalise sur l'emploi abstrait, avec ou sans complément circonstanciel - «Un peu de discrétion ne messiérait pas (Arnoux, Roi, 1956, p. 63)». Et le dernier évoque, par un extrait tiré de Balzac («Le menton en l'air à la Mirabeau est une attitude de fierté qui, selon moi, messied généralement (Balzac, Théor. démarche, 1833, p. 635)) », la forme affirmative du verbe en question.

L'une des œuvres lexicographiques ci-dessus mentionnées (TLFi) codifie, outre l'infinitif, une autre unité lexicale issue du paradigme de conjugaison du verbe en question : messéant,-ante. Nous tenons à souligner que cette lexie est absente du dictionnaire Le Petit Robert 2014, ce qui nous sert de justification approfondie pour les constatations esquissées dans l'introduction concernant les généralités de fonctionnement des verbes défectifs dans la langue française (voir supra). Nous ne nous permettons pas d'accuser les auteurs de cette œuvre lexicographique de négligence ou de retard, car comme l'explique Alain Rey (Le Petit Robert 2014 : XI) la macrostructure d'un dictionnaire "se construit sur un axe de fréquence, du plus courant au moins courant ». Grâce à la requête effectuée dans Frantext, au mois de septembre 2014, nous pouvons confirmer la légitimité de cette exclusion. Non seulement, en lançant les recherches sur toutes les formes conjuguées du verbe en question à l'aide de \&cmesseoir, le moteur de recherche 
n'a trouvé aucune apparition de son paradigme de conjugaison au sein de tous les textes postérieurs à l'an 2000, mais en plus, notre tentative de vérifier le fonctionnement correct de cet outil d'exploration textuelle a été inutile, car une nouvelle requête portant cette fois-ci sur l'adjectif \&mmesséant est restée de nouveau sans aucun résultat.

En consultant le TLFi en ligne, nous avons trouvé trois informations explicites sur la reconnaissance et la compréhension de ce signe lexical. Après l'indication qu'il s'agit d'un adjectif vieilli et littéraire, le TLFi montre l'emploi à l'écrit de cette unité lexicale par deux extraits dont les datations sont relativement anciennes : l'une remonte à l'année 1866 et l'autre, plus récente, est de 1910. Suit l'exemplification pour la tournure messéant à + substantif, avec renvoi à un extrait de 1863. En dernier lieu, la description traite la construction impersonnelle c'est messéant/il est messéant de + infinitif. Cette remarque contient un exemple ad illustrandum, tiré d'une source littéraire qui est la plus récente de toutes les références bibliographiques indiquées, même si elle n’est que de l'année 1939.

La description lexicographique du TLFi n'omet pas de rappeler que cet ancien participe présent qui, suite à une conversion, fonctionnait en français comme adjectif, a été remplacé par l'antonyme usuel de bienséant - malséant. L'adjectif malséant y figure comme un mot qui n'est pas d'usage familier. La valeur sémantique de cette unité lexicale est présentée par deux acceptions majeures : " contraire à la bienséance » et qui « sied mal en certaines circonstances ». Sa collocabilité affecte les animés ainsi que les inanimés et s'avère de gamme moyenne. Les cooccurrences observées dans les extraits tirés de Frantext (\&mmalséant) se prêtent au postulat confirmant le remplacement de l'emploi de cet adjectif. Ayant une valeur sémantique qui équivaut à l'addition de l'acception de chaque élément lexical, les combinaisons de cette lexie, que nous reproduisons ci-dessous, sont les manifestations de la combinabilité libre dans la langue française :

E095, le rapport sexuel avec la vieille femme, acte malséant entre tous encore aujourd'hui, en dépit de l'évolution

R210 que soit votre état réel, dont il serait franchement malséant de faire, justement, état. De toutes façons, là-bas E064 les Juifs de la manière la plus grossière et la plus malséante; d'autres résistaient mal à la tentation d'abuser E092 une tuméfaction vilaine; sous ce masque de croûtes malséantes, le mufle d'un jeune loup aux abois. - Sur les grandes

Dans l'optique des résultats obtenus, il n'est pas possible de confirmer que le verbe messeoir au sens de "n'être pas convenable " « s'emploie, [comme] dit l'Acad., dans les mêmes temps que seoir « convenir » (Grevisse 2001 : 1245). Les attestations dans l'ensemble des textes postérieurs à l'an 2000, dans le corpus électronique Frantext, ne renvoient qu'à la vivacité de l'adjectif malséant, construit, de manière analogique, à l'aide de trois morphèmes lexicaux, comme son antonyme bienséant. 


\section{Conclusion}

Classifié dans les ressources lexicographiques récemment publiées (Petit Robert 2014, TLFi, Larousse 2009) comme un verbe vieilli, le profil combinatoire de seoir, repéré dans la base textuelle Frantext dans des sources écrites postérieures à l'an 2000, confirme son maintien dans le vocabulaire du français contemporain, bien que son usage soit surtout réservé au domaine littéraire. Le moteur de recherche a trouvé, en fonction des critères imposés à la taille du corpus, 61 modes, temps et personnes du verbe seoir. Par ordre décroissant du nombre d'apparitions, il s'agissait de sied (14), seyant (12), sis (10), à part égale avec sise (10), seyait (6), séant (5) et, pour trois tiroirs verbaux, le taux de fréquence était identique : une seule attestation pour la troisième personne du pluriel de l'indicatif de l'imparfait, une pour la troisième personne du singulier du futur simple et une pour le conditionnel du présent.

Suite à l'extraction et au traitement de données ci-dessus décrits, nous pouvons constater la prédominance de l'emploi de la troisième personne du singulier de l'indicatif du présent (il sied : 22,95\%) du verbe seoir au sens de « convenir » et du participe passé variant en nombre et en genre (sis, sise, sises : 32,79\%) qui marque la valeur sémantique privilégiée "situé » et dont les configurations actancielles sont influencées par des facteurs discursifs variés.

Les autres temps (l'imparfait, le futur simple et le conditionnel présent) sont d'une manière égale usités aux troisièmes personnes, ce qui confirme l'exactitude de la nomenclature dictionnairique. Le taux de fréquence de l'emploi des troisièmes personnes dépasse ainsi un tiers $(37,7 \%)$ de toutes les formes conjuguées, présentes dans 152 textes indiqués.

En guise de conclusion, nous pouvons affirmer qu'à l'aide des requêtes effectuées par l'outil d'exploration textuelle Frantext, nous avons tracé le profil lexical et syntaxique des lexies choisies et dressé leur liste de cooccurrences lexicales dans le cadre des textes réunis. Hormis les blocs lexicaux tout faits (Frath, Gledhill 2005 : 12) fournis par les dictionnaires, le profil combinatoire du verbe défectif seoir est relativement varié et quelques modes, temps et personnes sont censés rester usités en français contemporain. Par contre, la capacité combinatoire du verbe défectif messeoir, attestée dans le corpus électronique Frantext, est de gamme restreinte et prouve un potentiel peu productif dans le mode d'expression écrite de la langue française contemporaine, ce qui lui attribue à juste titre l'étiquette de verbe défectif.

\section{Références bibliographiques}

Frantext. <http://www.frantext.fr>

Frath, P., \& Gledhill, Ch. (2005). Qu'est-ce qu’une unité phraséologique? In C. Bolly, J.-R. Klein, \& B. Lamirov (Eds.), La Phraséologie dans tous ses états. Cahiers de l'Institut de Linguistique de Louvain, 31(2-4), 11-25.

Grevisse, M. (2001). Le bon usage. Paris: DUCULOT.

Larousse. <http://www.larousse.fr/>

Le Petit Robert. (2014). Paris: Larousse. 
Mejri, S. (2008). La place du figement dans la description des langues. In Les séquences figées : entre langue et discours (pp. 117-129). Stuttgart: Franz Steiner Verlag.

Schapira, Ch. (1999). Les stéréotypes en français. Proverbes et autres formules. Paris: Ophrys.

Trésor de la langue française informatisé. <http://www.tlf.fr>.

Tutin, A, \& Grossmann, F. (2002). Collocations régulières et irrégulières: esquisse de typologie du phénomène collocatif. Revue française de linguistique appliquée, 7(1), 7-25. 
